\title{
ON THE EXISTENCE OF FUNDAMENTAL SOLUTIONS OF CERTAIN BOUNDARY VALUE PROBLEMS
}

\author{
R. S. PATHAK
}

\begin{abstract}
An existence theorem for fundamental solutions of a large class of boundary value problems involving the Bessel type differential operator $S_{\mu}$ is proved. The problem includes the Cauchy problem of the differential operator $S_{\mu}$ as a special case.
\end{abstract}

1. Barros-Neto [1] has given fundamental solutions of the boundary value problem in a half space with constant coefficients. The problem can be described in brief as follows:

Let $P\left(D, D_{t}\right)$ be a hypoelliptic partial differential operator and let $Q_{1}(D$, $\left.D_{t}\right), \ldots, Q_{m}\left(D, D_{t}\right)$ be given partial differential operators in $R^{n+1}$ with constant coefficients, where

$$
D=\left(D_{1}, \ldots, D_{n}\right), \quad D_{j}=(1 / i)\left(\partial / \partial x_{j}\right), \quad D_{t}=(1 / i)(\partial / \partial t),
$$

and $m$ is the number of roots with positive imaginary parts of the characteristic polynomial in $\tau, P(\xi, \tau)=0$. Then the solution of the boundary value problem defined by the operators $\left(P\left(D, D_{t}\right), Q_{1}\left(D, D_{t}\right), \ldots, Q_{m}\left(D, D_{t}\right)\right)$ in the half space $R_{+}^{n+1}=\left\{(x, t): x \in R^{n}, t>0\right\}$ are certain tempered distributions.

In this paper we are interested in the solutions of another class of boundary value problems. Let $S_{\mu}$ denote the differential operator $d^{2} / d x^{2}+(1-$ $\left.4 \mu^{2}\right) / 4 x^{2}, \mu>-\frac{1}{2}$. Then by using the theory of distributional Hankel transforms ([6] and [9]), we show that the solutions of the boundary value problem defined by $\left(P\left(S_{\mu}, D_{t}\right), Q_{1}\left(S_{\mu}, D_{t}\right), \ldots, Q_{m}\left(S_{\mu}, D_{t}\right)\right)$ are certain generalized functions belonging to $H_{\mu}^{\prime}$ [6].

2. The main results are contained in the following:

THEOREM. There are distributions $K(x, t), K_{1}(x, t), \ldots, K_{m}(x, t)$ in $H_{\mu}^{\prime}\left(R_{+}^{n+1}\right)$ such that $K, K_{1}, \ldots, K_{m}$ are infinitely differentiable functions in $R_{+}^{n+1}$ which can be extended to infinitely differentiable functions in ${\overline{R_{+}^{n+1}}}_{-}^{n}\{0\}$. If we keep the same notation for the extended functions, then $K(x, t)$ satisfies, in the sense of distributions, the boundary problem

Received by the editors October 27, 1977.

AMS (MOS) subject classifications (1970). Primary 35A10; Secondary 44A15.

Key words and phrases. Fundamental solutions, boundary value problems, Cauchy problem, differential operator $S_{\mu}$, Hankel transformation, tempered distributions.

(c) 1979 American Mathematical Society $0002-9939 / 79 / 0000-0061 / \$ 02.50$ 


$$
\begin{aligned}
P\left(S_{\mu}, D_{t}\right) K & =\delta(x-1) \otimes \delta(t)-\alpha(x) \otimes \delta(t) \quad \text { in } \overline{R_{+}^{n+1}}, \\
\left.Q_{\nu}\left(S_{\mu}, D_{t}\right) K\right|_{R_{0}^{n}} & =0, \quad 1 \leqslant \nu \leqslant m,
\end{aligned}
$$

while every $K_{l}(x, t)$ satisfies

$$
\begin{aligned}
P\left(S_{\mu}, D_{t}\right) K_{l} & =0 \quad \text { in } R_{+}^{n+1}, \\
\left.Q_{\nu}\left(S_{\mu}, D_{t}\right) K_{l}\right|_{R_{0}^{n}} & =\delta_{\nu, l}(\delta(x-1)-\alpha(x)), \quad 1<\nu<m,
\end{aligned}
$$

where $\delta_{\nu, l}$ is the Kronecker delta, $\delta$ is the Dirac measure, $\alpha(x) \in H_{\mu}\left(R^{n}\right)$ and

$$
\left.Q_{\nu} K\right\rceil_{R_{0}^{n}}=\lim _{t \rightarrow 0^{+}} Q_{\nu} K(x, t)
$$

the limit being taken in $H_{\mu}^{\prime}\left(R^{n}\right)$.

PROOF. For each $\xi \in R^{n}$, let $\tau_{1}(\xi), \ldots, \tau_{m}(\xi)$ be the roots of the characteristic polynomial $P(\xi, \tau)=0$ with positive imaginary parts. Let

$$
K_{\xi}(\tau)=\prod_{j=1}^{m}\left(\tau-\tau_{j}(\xi)\right)
$$

and define the characteristic function $C(\xi)$ by

$$
\begin{array}{rl}
C(\xi)=R\left(K_{\xi} ; Q_{1}, \ldots, Q_{m}\right)=\frac{\operatorname{det} Q_{\nu}\left(\xi, \tau_{j}(\xi)\right)}{\Pi_{k<j}\left(\tau_{j}(\xi)-\tau_{k}(\xi)\right)}, & \\
1 & 1 \leqslant \nu \leqslant m, 1 \leqslant j \leqslant m .
\end{array}
$$

Then $C(\xi)$ is a polynomial in $\xi$ and according to Hörmander [4] there exists a constant $M>0$ such that for all $\xi \in R^{n}$ with

$$
|\xi|>M, \quad C(\xi) \neq 0 .
$$

Next, for $\nu=1,2, \ldots, m, t>0$ and for all $\xi$ satisfying $|\xi|>M$ define the function $H_{\nu}(\xi, t)$ by

$$
H_{\nu}(\xi, t)=\frac{R\left(K_{\xi} ; Q_{1}(\xi, \tau(\xi)), \ldots, e^{i t \tau(\xi)}, \ldots, Q_{m}(\xi, \tau(\xi))\right.}{C(\xi)}
$$

where

$$
R\left(K_{\xi} ; Q_{1}(\xi, \tau(\xi)), \ldots, e^{i t \tau(\xi)}, \ldots, Q_{m}(\xi, \tau(\xi))\right)
$$

indicates that in the determinant appearing in (3) the $\nu$ th row has been replaced by $\left(e^{i t \tau_{1}(\xi)}, \ldots, e^{i t \tau_{m}(\xi)}\right)$. It is easily seen that $H_{\nu}(\xi, t), 1<\nu<m$, is a solution of the initial value problem

$$
\begin{aligned}
P\left(\xi, D_{t}\right) H_{\nu}(\xi, t) & =0, \\
Q_{l}\left(\xi, D_{t}\right) H_{\nu}(\xi, 0) & =\delta_{l, v}, \quad 1<l<m .
\end{aligned}
$$

Now, let us introduce a function $G_{0}(\xi, t)$ defined by

$$
G_{0}(\xi, t)=\frac{1}{2 \pi} \int_{-\infty}^{\infty} \frac{e^{i t \tau}}{P(\xi, \tau)} d \tau
$$

the integral being absolutely convergent if the degree of $P(\xi, \tau)$ in $\tau$ is $>2$ 
and convergent if it is equal to 1 . Obviously,

$$
P\left(\xi, D_{t}\right) G_{0}(\xi, t)=\delta(t)
$$

Next, for all $|\xi|>M$, set

$$
G(\xi, t)=G_{0}(\xi, t)-\sum_{\nu=1}^{m}\left(Q_{\nu}\left(\xi, D_{t}\right) G_{0}\right)(\xi, 0) H_{\nu}(\xi, t) .
$$

Then $G(\xi, t)$ is a solution of the initial value problem

$$
\begin{aligned}
P\left(\xi, D_{t}\right) G(\xi, t) & =\delta(t), \\
Q_{l}\left(\xi, D_{t}\right) G(\xi, 0) & =0, \quad 1<l<m,
\end{aligned}
$$

for all $|\xi|>M$. Assume that $M>0$ is such that when $|\xi|>M, G$ and $H_{\nu}$, $1<\nu<m$, are well defined. We define a new function $\chi(\xi) \in C_{c}^{\infty}\left(R^{n}\right)$ by

$$
\begin{aligned}
\chi(\xi) & =1, & & |\xi|<M, \\
& =0, & & |\xi|>M+1 .
\end{aligned}
$$

Then following the technique of Barros-Neto [1] and using the fact that $\left|y_{i}^{1 / 2} J_{\mu}\left(y_{i}\right)\right|<C$ for all $y_{i}>0$ and $\mu>-\frac{1}{2}$, we can prove that

$$
(1-\chi(y)) G\left(-y^{2}, t\right) \prod_{i=1}^{n} y_{i}^{1 / 2} J_{\mu}\left(y_{i}\right)
$$

and

$$
(1-\chi(y)) H_{\nu}\left(-y^{2}, t\right) \prod_{i=1}^{n} y_{i}^{1 / 2} J_{\mu}\left(y_{i}\right)
$$

are tempered distributions in $R^{n}$ which are concentrated on $\{y:|y|>M\}$ and therefore by the $n$-dimensional analogue of [9, Problem 5.2.3, p. 134] these are elements of $H_{\mu}^{\prime}$. Hence, we can define their generalized Hankel transforms $h_{\mu}^{\prime}$. Since for $\mu>-\frac{1}{2}, h_{\mu}^{\prime}$ is an automorphism on $H_{\mu}^{\prime}[6$, p. 430] the inverse Hankel transforms can also be defined. Consequently, we are justified in defining $K(x, t)$ and $K_{v}(x, t)$ by

$$
K(x, t) \triangleq h_{\mu, x}^{\prime}\left((1-\chi(y)) G\left(-y^{2}, t\right) \prod_{i=1}^{n} y_{i}^{1 / 2} J_{\mu}\left(y_{i}\right)\right)
$$

and

$$
K_{\nu}(x, t) \triangleq h_{\mu, x}^{\prime}\left((1-\chi(y)) H_{\nu}\left(-y^{2}, t\right) \prod_{i=1}^{n} y_{i}^{1 / 2} J_{\mu}\left(y_{i}\right)\right), \quad 1 \leqslant \nu \leqslant m
$$

These are also elements of $H_{\mu}^{\prime}$. Following the technique of Barros-Neto [1] it can be proved that these kernels satisfy (1) and (2) and they are $C^{\infty}$-functions in $\overline{R_{+}^{n+1}}-\{0\}$. 
Now, using the $n$-dimensional analogue of the results [9,pp. 143, 144]

$$
\begin{aligned}
h_{\mu}^{\prime}\left(-y^{2} f\right) & =S_{\mu} h_{\mu}^{\prime} f, \\
h_{\mu}^{\prime} \delta(x-1) & =\prod_{i=1}^{n} y_{i}^{1 / 2} J_{\mu}\left(y_{i}\right), \\
h_{\mu}^{\prime}\left[\prod_{i=1}^{n} x_{i}^{1 / 2} J_{\mu}\left(x_{i}\right)\right] & =\delta(y-1),
\end{aligned}
$$

and operating formally we have

$$
\begin{aligned}
P\left(S_{\mu}, D_{t}\right) K & =h_{\mu}^{\prime}\left((1-\chi(y)) P\left(-y^{2}, D_{t}\right) G\left(-y^{2}, t\right) \prod_{i=1}^{n} y_{i}^{1 / 2} J_{\mu}\left(y_{i}\right)\right) \\
& =h_{\mu}^{\prime}\left((1-\chi(y)) \prod_{i=1}^{n} y_{i}^{1 / 2} J_{\mu}\left(y_{i}\right)\right) \otimes \delta(t) \\
& =\delta(x-1) \otimes \delta(t)-\alpha(x) \otimes \delta(t)
\end{aligned}
$$

and

$$
\begin{aligned}
\lim _{t \rightarrow 0^{+}} Q_{\nu}\left(S_{\mu}, D_{t}\right) K \\
\quad=h_{\mu}^{\prime}\left((1-\chi(y)) P\left(-y^{2}, D_{t}\right) G\left(-y^{2}, 0\right)\right)=0, \quad 1 \leqslant \nu \leqslant m .
\end{aligned}
$$

Similarly we can derive the results (2).

Our theorem will be proved by justifying the above formal manipulations. For this, we need merely to prove the above limiting operations in $H_{\mu}^{\prime}$, which is the crucial part of the work. Let $\phi(x) \in H_{\mu}$; then we have to prove that

$$
\left\langle Q_{\nu}\left(S_{\mu}, D_{t}\right) K_{l}(x, t), \phi(x)\right\rangle \rightarrow\left\langle Q_{\nu}\left(S_{\mu}, D_{t}\right) K_{l}(x, 0), \phi(x)\right\rangle
$$

$$
(1<l<m)
$$

and

$$
\left\langle Q_{v}\left(S_{\mu}, D_{t}\right) K(x, t), \phi(x)\right\rangle \rightarrow\left\langle Q_{v}\left(S_{\mu}, D_{t}\right) K(x, 0), \phi(x)\right\rangle
$$

as $t \rightarrow 0^{+}$.

Now, in view of the definition (10), we have

$$
\begin{aligned}
\left\langle Q_{\nu}\left(S_{\mu}, D_{t}\right) K_{l}(x, t), \phi(x)\right\rangle \\
=\left\langle Q_{\nu}\left(S_{\mu}, D_{t}\right) h_{\mu}^{\prime}\left[(1-\chi(y)) H_{l}\left(-y^{2}, t\right) \prod_{i=1}^{n} y_{i}^{1 / 2} J_{\mu}\left(y_{i}\right)\right], \phi(x)\right\rangle \\
=\left\langle(1-\chi(y)) Q\left(-y^{2}, \tau\right) H_{l}\left(-y^{2}, t\right) \prod_{i=1}^{n} y_{i}^{1 / 2} J_{\mu}\left(y_{i}\right), \Phi(y)\right\rangle
\end{aligned}
$$

where $\Phi(y)=h_{\mu}[\phi(x)]$.

Expanding the determinant by means of which $H_{l}(\xi, t)$ has been defined, 
we have

$$
H_{l}(\xi, t)=\sum_{r=1}^{m} e^{i t \tau_{r}(\xi)} A_{\tau}^{r}(\xi) / C(\xi)
$$

where $A_{\tau}^{r}(\xi)$ is a polynomial in $\xi$. Hence the expression (13) can be written as

$$
\sum_{r=1}^{m}\left\langle(1-\chi(y)) Q_{\nu}\left(-y^{2}, \tau\right) \prod_{i=1}^{n} y_{i}^{1 / 2} J_{\mu}\left(y_{i}\right) A_{\tau}^{r}\left(-y^{2}\right) / C\left(-y^{2}\right), e^{i t \tau_{r}\left(-y^{2}\right)} \Phi(y)\right\rangle \text {. }
$$

In the following we shall show that, as $t \rightarrow 0^{+}, e^{i t_{r}\left(-y^{2}\right)} \Phi(y)$ converges in $H_{\mu}$ to $\Phi(y)$. Then the limiting operation (11) will follow from this result and the fact that

$$
(1-\chi(y)) Q_{\nu}\left(-y^{2}, \tau\right) \prod_{i=1}^{n} y_{i}^{1 / 2} J_{\mu}\left(y_{i}\right) \frac{A_{\tau}^{r}\left(-y^{2}\right)}{C\left(-y^{2}\right)} \in H_{\mu}^{\prime} .
$$

According to an $n$-dimensional analogue of [9, see 5.3] our above assertion will be established as soon as we prove that as $t \rightarrow 0^{+}$,

$$
\frac{e^{i t \tau_{r}\left(-y^{2}\right)}}{1+y^{n_{p}}} \rightarrow \frac{1}{1+y^{n_{p}}}
$$

for some positive integer $n_{\nu}$ and, for each positive integer $\nu$,

$$
\frac{\left(y^{-1} D_{y}\right)^{\nu}}{1+y^{n_{2}}} e^{i t \tau_{r}\left(-y^{2}\right)} \rightarrow 0 \text { as } t \rightarrow 0^{+}
$$

uniformly for all $y \in R^{n}-\{0\}$.

Notice that

$$
\left(y^{-1} D_{y}\right)^{\nu} e^{i t \tau_{r}\left(-y^{2}\right)}=2^{\nu}\left[D_{u}^{\nu} e^{i t \tau_{r}(-u)}\right]_{u=y^{2}}
$$

The differentiation of $e^{i t \tau_{r}(-u)}$ with respect to $u$ yields a finite number of terms which are of the form

$$
A_{r} t^{p} \tau_{r}^{(q)}(-u) e^{i t \tau_{r}(-u)}
$$

Here $\tau_{r}^{(q)}$ denotes $(d / d y)^{q} \tau_{r}(-y)$. Since all the roots of $P(\xi, \tau)=0$ satisfy the inequality $[2$, p. 518$]$

$$
|\tau(\xi)| \leqslant A\left(|\xi|^{B}+1\right)
$$

and by Lemma 4 of [2],

$$
\left|D_{\xi}^{q} \tau(\xi)\right| \leqslant A_{q}^{\prime}|\xi|^{B+|q|}\left(\sum_{j=1}^{n}\left|\xi_{j}\right|^{1 / d_{j}}\right)^{-|q|}
$$

for all $\xi \in R^{n}$ such that $\sum_{j=1}^{n}\left|\xi_{j}\right|^{1 / d_{j}} \geqslant M+1$, we have

$$
\left|t^{p} \tau_{r}^{(q)} e^{i t \tau_{r}(-u)}\right| \leq C t^{p}|u|^{C^{\prime}}
$$

for certain finite constants $C$ and $C^{\prime}$. Therefore, choosing $n_{\nu}>2 C^{\prime}$, we have

$$
\frac{\left(y^{-1} D_{y}\right)^{\nu}}{1+y^{n_{2}}} e^{i t \tau_{r}\left(-y^{2}\right)} \rightarrow 0 \text { as } t \rightarrow 0,
$$

uniformly for all $y \in R^{n}-\{0\}$. 
Further to prove (14), we first note that, given $\varepsilon>0$, there exists an $R<\infty$, such that, for all $y>R$ and $t \geqslant 0$,

$$
0<\frac{\left|1-e^{i t \tau_{r}\left(-y^{2}\right)}\right|}{1+y^{n_{3}}} \leqslant \frac{1+e^{-t\left|\operatorname{Im} \tau_{r}\left(-y^{2}\right)\right|}}{1+R^{n_{3}}}<\frac{2}{1+R^{m_{1}}}<\varepsilon .
$$

Having fixed $R$ this way, we restrict $y$ to $0<y<R$ and then write

$$
\begin{aligned}
0 & \leqslant \frac{\left|1-e^{i t \tau_{r}\left(-y^{2}\right)}\right|}{1+y^{n_{2}}} \leqslant \frac{\int_{0}^{t}\left|\tau_{r}\left(-y^{2}\right)\right| e^{-s \operatorname{Im} \tau_{r}\left(-y^{2}\right)} d s}{1+y^{m_{2}}} \\
& \leqslant \frac{t\left|\tau_{r}\left(-y^{2}\right)\right|}{1+y^{n_{3}}}<C \frac{t\left(|y|^{2 d}+1\right)}{1+y^{n_{2}}}
\end{aligned}
$$

by (16). Choosing $n_{v}>2 d$, we see that the last expression tends to zero as $t \rightarrow 0^{+}$uniformly for all $y \in R^{n}$. Therefore, there exists a $T>0$ such that, for all $0<t<T$,

$$
0 \leqslant \frac{\left|1-e^{i t \tau_{r}\left(-y^{2}\right)}\right|}{1+y^{n_{3}}} \leqslant \varepsilon, \quad y \in R^{n} .
$$

Since $\varepsilon$ is arbitrary our assertion is proved.

\section{REFERENCES}

1. J. Barros-Neto, The parametrix of a regular hypoelliptic boundary value problem, Ann. Scuola Norm. Sup. Pisa Fasc. I 26 (1972), 247-268.

2. __, On regular hypoelliptic boundary value problems, J. Math. Anal. Appl. 14(1973), $508-530$.

3. I. M. Gel'fand and G. E. Silov, Generalized functions, vol. 3: Some questions on the theory of differential equations, Academic Press, New York, 1967.

4. L. Hörmander, On the regularity of the solutions of boundary problems, Acta Math. 99 (1958), 225-264.

5. __ Linear partial differential operators, Springer-Verlag, Berlin, 1963.

6. E. L. Koh, The n-dimensional distributional Hankel transformation, Canad. J. Math. 17 (1975), 423-433.

7. W. Y. Lee, On the Cauchy problem of the differential operator $S_{\mu}$, Proc. Amer. Math Soc. 51 (1975), 149-154.

8. L. Schwartz, Théorie des distributions. vols. 1, 2, Hermann, Paris, 1957, 1959.

9. A. H. Zemanian, Generalized integral transformations, Interscience, New York, 1968.

Departmient of Mathematics, Banaras Hindu University, Varanasi 221005, India 